\title{
Adult population as potential reservoir of NTD infections in rural villages of Kwale district, Coastal Kenya: implications for preventive chemotherapy interventions policy
}

Sammy M Njenga ${ }^{1 *}$, Charles S Mwandawiro ${ }^{1}$, Erastus Muniu² ${ }^{2}$ Mariam T Mwanje ${ }^{3}$, Fatma M Haji ${ }^{4}$ and Moses J Bockarie

\begin{abstract}
Background: Neglected tropical diseases (NTDs) are major public health problems in developing countries where they contribute to suffering of populations living in poor settings. As part of a research project started in September 2009 in Kwale district, Coast Region, Kenya, a baseline cross-sectional survey was conducted in 5 rural villages to provide information on the status of NTDs, including urinary schistosomiasis, soil-transmitted helminthiasis (STH), and lymphatic filariasis. This paper presents the results of a parasitological investigation among adults in the study villages.
\end{abstract}

Methods: A total of 599 adults in the 5 study villages were tested for NTD infections in urine, stool and blood. The presence of Schistosoma haematobium infection was determined by the urine filtration method. The presence of STH in stool was determined by Kato-Katz method while filarial antigenaemia was determined using immunochromatographic (ICT) test.

Results: The study revealed high prevalence of hookworm (41.7\%) and schistosomiasis (18.2\%) infections among adults in the study villages. Of the 599 individuals examined, $50.1 \%$ had one or more helminthic infections. There was low level of polyparasitism with helminthic NTDs in the study population with $9.5 \%$ and $1.7 \%$ of the participants having two and three infections, respectively.

Conclusions: In the current study, hookworm and schistosomiasis infections were identified as important infections among adults living in areas of high endemicity for these infections. Thus, if this section of the population is left untreated it may remain an important potential reservoir and a source of re-infection for school-age children treated in school deworming programmes. Therefore, there is a need to design novel strategies for preventive chemotherapy interventions that could allow inclusion of adults in an effort to reduce force of infection in high endemic communities.

\section{Background}

Neglected tropical diseases (NTDs) include seven helminth infections namely soil-transmitted helminths $(\mathrm{STH})$ infections (hookworm, ascariasis, trichuriasis), lymphatic filariasis (LF), onchocerciasis, guinea worm disease (dracunculiasis), and schistosomiasis [1]. Soil-

\footnotetext{
* Correspondence: sammynjenga@gmail.com

${ }^{1}$ Eastern and Southern Africa Centre of International Parasite Control (ESACIPAC), Kenya Medical Research Institute (KEMRI), Mbagathi Road, Nairobi, Kenya

Full list of author information is available at the end of the article
}

transmitted helminths $(\mathrm{STH})$ and schistosomiasis are mostly prevalent in developing countries due to poor sanitation and lack of adequate clean water. Soil-transmitted helminth infections are among the most prevalent infections in the world with around 1.4 billion individuals infected by Ascaris lumbricoides, 1.0 billion by Trichuris trichiura, and 1.3 billion by hookworms [2]. Of an estimated 200 million people infected with schistosomiasis, 170 million live in sub-Saharan Africa while the remaining 30 million live in North Africa, Asia and South America

\section{Ciomed Central}


[3]. Lymphatic filariasis is a major cause of debilitating chronic disability and is estimated to infect over 120 million people worldwide [4]. The coastal areas of Kenya are highly endemic for urinary schistosomiasis, STH, and LF [5-7]. Children have the highest prevalence and intensity of STH and schistosomiasis infections, but the consequences of chronic infection, such as growth stunting, anaemia, hepatic/urinary fibrosis, and impaired cognitive development, continue to have an effect throughout adulthood [8].

For a long time most national health authorities in sub-Saharan Africa have shown little commitment to control of NTDs mainly due to lack of resources [9]. However, the situation is now changing with the realization that deworming is a relatively simple, effective, and cost-effective public health measure that can be readily integrated with existing health care programmes. This has led to renewed interest in control of parasitic diseases; in 1997, the $50^{\text {th }}$ World Health Assembly (WHA) passed a resolution to eliminate LF globally as a public health problem [10]. Following the WHA resolution on LF elimination, the World Health Organization (WHO) launched the Global Programme to Eliminate Lymphatic Filariasis (GPELF) in 2000 [11]. Subsequently in 2001, during the $54^{\text {th }}$ WHA, the WHO urged its member states to ensure provision for the regular anthelminthic treatment of all school-age children living in areas of schistosomiasis and STH endemicity [2]. Targeting anthelminthics to school-age children capitalizes on the fact that the heaviest burdens of infection are found in this segment of the population. In 2009, a research project to evaluate an alternative strategy for controlling morbidity due to schistosomiasis and STH and its integration with the national LF elimination programme was established in Kwale district, Coast Region, Kenya. The current study was conducted to assess the levels of schistosomiasis, STH and LF infections in adults living in 5 rural villages selected for the research project.

\section{Methods}

\section{Study site and design}

This cross-sectional study was conducted in January 2010 in 5 villages in Mwaluphamba location, Kwale district, Coast Region, Kenya. Kwale district is in Kwale County and covers an area of $1,043 \mathrm{~km}^{2}$ which is divided into two administrative divisions, namely Matuga and Kubo divisions: the study area is situated in Kubo division. The populations of Kwale County and Kwale district based on 2009 census were estimated to be 649,931 and 151,978 persons, respectively. In terms of climate, the district is hot and dry from January to March and relatively cool from June to August. Rainfall pattern is bimodal with long rains normally occurring between mid-March and June while the short rains occur between October and December.

Subsistence farming of food crops including maize, cowpeas and cassava is done mainly for domestic consumption. Coconuts, oranges, mangoes and cashew nuts are also grown for both domestic consumption and as cash crops. The main livestock kept include cattle, goats, ducks and chickens. The protocol for this study was reviewed and approved by the Scientific Steering and Ethical Review Committees of the Kenya Medical Research Institute (KEMRI). Oral informed consent was obtained from study participants.

\section{Sample collection and examination}

Convenience samples of approximately 150 adults in each village were recruited to participate in the study on a voluntary basis. Stool containers were given to study participants the day before sample collection. Collection of samples was done the following morning at designated places (usually schools) agreed at meetings held in each village, but there were some people who did not turn up for sample collection. Samples collected included stool, urine, and $3 \mathrm{ml}$ venous blood. The samples were transported to KEMRI field station laboratory located at the Kwale district hospital for processing.

All samples were obtained between 10:00 am. and 1:00 $\mathrm{pm}$. Urine samples were collected in wide-mouth plastic containers. Immediately the urine samples were collected, $10 \mathrm{ml}$ specimens were used for examination of schistosome eggs using urine filtration technique. Briefly, the $10 \mathrm{ml}$ urine specimens were aliquoted using disposal syringes and filtered through $12 \mu \mathrm{m}$ polycarbonate membrane filters at the sample collection site. The filters were carefully placed on labeled microscope glass slides and stored in slide boxes for later reading under a light microscope. Additional $5 \mathrm{ml}$ aliquots of urine specimens preserved with sodium azide $(0.1 \% \mathrm{w} / \mathrm{v})$ were collected for later assessment of morbidity due to $S$. haematobium infection.

Lymphatic filariasis infection was assessed in $100 \mu \mathrm{l}$ venous blood aliquots using the immunochromatographic (ICT) test which detects circulating filarial antigens. Thick and thin blood smears were made on clean microscope slides and air-dried before storage in slide boxes. The thin smear was fixed with methanol and then both smears stained with 10\% Giemsa stain, pH 7.0 for $10 \mathrm{~min}$. The smears were rinsed briefly in PBS buffer before drying at room temperature. The thick smear was used to examine for malaria parasites under oil immersion objective and the thin film used to determine species for positive slides. The remaining blood specimens were used for preparation of plasma samples to be used later for serological assays related to the research project. The plasma and urine specimens were stored at 
$4^{\circ} \mathrm{C}$ while at the KEMRI field station and frozen at $-80^{\circ} \mathrm{C}$ upon return to central laboratories in Nairobi.

The quantitative Kato-Katz method was used to examine the stool samples for presence of different intestinal helminth species eggs as previously described. Briefly, two $41.7 \mathrm{mg}$ stool smears were made from each stool sample on the day of sample collection for examination and counting of STH eggs. The smears were read within 1 hour for hookworm eggs and next day for other STH eggs. Egg counts were expressed as eggs per gram of stool (EPG). Individuals were categorized as having light- or heavy-intensity infections based on the criteria of the WHO [12]. For S. haematobium, light-intensity infections were 1 - 49 eggs/10 $\mathrm{ml}$ urine and heavy-intensity infections $\geq 50$ eggs $/ 10 \mathrm{ml}$ urine. Hookworm infections were classified as light-intensity when counts were1-1,999 EPG and heavy-intensity when counts were $\geq 2000$ EPG.

\section{Data management and analysis}

Data were double-entered into a computer using MS Access and after validation analyzed using SPPS 12.0 statistical analysis software (SPSS Inc., Chicago, Illinois, USA). Chi-square $\left(\mathrm{X}^{2}\right)$ test was used to compare proportions between the villages. One-way Analysis of Variance (ANOVA) was used to compare differences between group means and Kruskal-Wallis one-way ANOVA to compare group medians. A value of $\mathrm{P}<0.05$ was considered statistically significant.

\section{Results}

Demographic characteristics of the study group are summarized in Table 1. A greater number of female participants were examined compared to males ( $\mathrm{p}<0.01$ ). Additionally, female participants were significantly younger (median age 35 years, range 15 - 88 years) compared to male participants (median age 46 years, range 18 - 85 years $)$; $(\mathrm{P}<0.001)$.

Prevalence of five helminthic infections and malaria among the study villages are summarized in Table 2.

Table 1 Demographic characteristics of study participants in five villages, Kwale district, coastal Kenya

\begin{tabular}{lllllll}
\hline Characteristic & Maponda & Mirihini & Kajiweni & Miatsani & Mlafyeni & All \\
\hline No. tested & 116 & 125 & 122 & 93 & 137 & 593 \\
*Female & 77 & 94 & 69 & 70 & 99 & 409 \\
Male & 39 & 31 & 53 & 23 & 38 & 187 \\
$\begin{array}{l}\text { Median age } \\
\text { (yrs) }\end{array}$ & 38 & 33 & 40 & 41 & 38 & 38 \\
$\begin{array}{l}\text { Age range } \\
\text { (yrs) }\end{array}$ & $18-85$ & $18-80$ & $15-83$ & $18-82$ & $18-88$ & $15-$ \\
\hline
\end{tabular}

*There were more female participants and they were significantly younger compared to male participants $(P<0.001)$. Data on sex/age for 6 participants were missing.
There was high prevalence of hookworm (41.7\%) and schistosomiasis (18.2\%) infections among adults in the study villages. Of 107 persons with S. haematobium infection, 49 (45.8\%) had hookworm infection, 2 (1.9\%) had $T$. trichiura, 7 (6.7\%) had LF, and 6 (5.7\%) had malaria. The general prevalence of malaria in the study area was $5.6 \%$ and except for one village (Miatsani) malaria was prevalent in all other four villages.

There was no significant difference in prevalence of STH and schistosomiasis infections between males and females. The prevalence of LF infection, however, was significantly higher in males $(8.2 \%)$ than in females $(2.2 \%)$; $(\mathrm{p}<0.01)$. Intensities of hookworm and schistosomiasis infections among adults positive for these infections in the 5 study villages are summarized in Table 3. The majority of these infections were lightintensity and there were no statistically significant differences among the 5 villages. Overall, > 95\% of hookworm and $>75 \%$ of schistosomiasis infections were categorized as light-intensity. Additionally, Ascaris and Trichuris infections were light-intensity.

The status of polyparasitism in the study area is summarized in Table 4 . Overall, $50.1 \%$ of the study population had one or more helminthic infections. There was low level of polyparasitism with helminthic NTDs in the study population with $9.5 \%$ and $1.7 \%$ of the participants having two and three infections, respectively.

\section{Discussion}

Few studies have been conducted to quantify the extent of schistosomiasis and STH infections among adults living in areas of high endemicity for these infections. Data from this study revealed a relatively high prevalence of $S$. haematobium and hookworm infections in the adult population. Current WHO guidelines recommend preventive chemotherapy based on regular anthelminthic drugs as a public health intervention to control helminthic infections and reduce morbidity [13]. Many public health programmes targeting STH and schistosomiasis currently employ school-based deworming which has been previously shown to provide externality benefits to untreated groups within and close to the treatment schools [14]. The results of our study, however, suggest that if adults are left untreated in areas where prevalence of helminthic infections is high in this group, the adults may act as a reservoir for transmission and a source of reinfections to the school-age children and thus present an obstacle to the control programmes.

On average, around $4 \%$ of the adults in this study had circulating filarial antigen (CFA). By the time this study was conducted, Kwale district had received three rounds of diethylcarbamazine citrate (DEC)/albendazole mass drug administration (MDA) against LF (in September 2003, March 2005, and December 2008) under the 
Table 2 Prevalence of urinary schistosomiasis, STH, LF and malaria among study participants in five rural villages in Kwale district, coastal Kenya

\begin{tabular}{|c|c|c|c|c|c|c|}
\hline \multirow[b]{2}{*}{ Village } & \multicolumn{5}{|c|}{$\begin{array}{l}\text { No. infected/No. examined (\%) } \\
95 \% \mathrm{Cl}(\%)\end{array}$} & \multirow[b]{2}{*}{ Malaria } \\
\hline & Ascaris & Trichuris & Hookworm & Schisto & LF & \\
\hline Maponda & $\begin{array}{l}0 / 106(0) \\
-\end{array}$ & $\begin{array}{l}4 / 106(3.8) \\
0.2-7.4\end{array}$ & $\begin{array}{l}40 / 105(38.1) \\
28.8-47.4\end{array}$ & $\begin{array}{l}10 / 117(8.5) \\
3.4-13.5\end{array}$ & $\begin{array}{l}6 / 118(5.1) \\
1.1-9.1\end{array}$ & $\begin{array}{l}9 / 118(7.6) \\
2.8-12.4\end{array}$ \\
\hline Mirihini & $\begin{array}{l}0 / 121(0) \\
-\end{array}$ & $\begin{array}{l}0 / 122(0) \\
-\end{array}$ & $\begin{array}{l}58 / 119(48.7) \\
39.7-57.7\end{array}$ & $\begin{array}{l}18 / 127(14.2) \\
8.1-20.3\end{array}$ & $\begin{array}{l}4 / 126(3.2) \\
0.1-6.3\end{array}$ & $\begin{array}{l}4 / 127(3.1) \\
0.1-6.1\end{array}$ \\
\hline Kajiweni & $\begin{array}{l}2 / 122(1.6) \\
0-3.8\end{array}$ & $\begin{array}{l}1 / 122(0.8) \\
0-2.4\end{array}$ & $\begin{array}{l}64 / 120(53.3) \\
44.4-62.2\end{array}$ & $\begin{array}{l}25 / 122(20.5) \\
13.3-27.7\end{array}$ & $\begin{array}{l}7 / 122(5.7) \\
1.6-9.8\end{array}$ & $\begin{array}{l}12 / 120(10.0) \\
4.6-15.4\end{array}$ \\
\hline Miatsani & $\begin{array}{l}0 / 92(0) \\
-\end{array}$ & $\begin{array}{l}1 / 92(1.1) \\
0-3.2\end{array}$ & $\begin{array}{l}44 / 93(47.3) \\
37.2-57.4\end{array}$ & $\begin{array}{l}20 / 93(21.5) \\
13.2-29.8\end{array}$ & $\begin{array}{l}4 / 91(4.4) \\
0.2-8.6\end{array}$ & $\begin{array}{l}0 / 93(0) \\
-\end{array}$ \\
\hline Mlafyeni & $\begin{array}{l}2 / 129(1.6) \\
0-3.8\end{array}$ & $\begin{array}{l}0 / 129(0) \\
-\end{array}$ & $\begin{array}{l}29 / 127(22.8) \\
15.5-30.1\end{array}$ & $\begin{array}{l}34 / 128(26.6) \\
18.9-34.3\end{array}$ & $\begin{array}{l}4 / 137(2.9) \\
0.1-5.7\end{array}$ & $\begin{array}{l}8 / 134(6.0) \\
2.0-10.0\end{array}$ \\
\hline All & $\begin{array}{l}4 / 570(0.7) \\
0-1.4\end{array}$ & $\begin{array}{l}6 / 571(1.1) \\
0.2-2.0\end{array}$ & $\begin{array}{l}235 / 564(41.7) \\
37.6-45.8\end{array}$ & $\begin{array}{l}107 / 587(18.2) \\
15.1-21.3\end{array}$ & $\begin{array}{l}25 / 594(4.2) \\
2.6-5.8\end{array}$ & $\begin{array}{l}33 / 592(5.6) \\
3.7-7.5\end{array}$ \\
\hline *P value & & & $<0.001$ & 0.030 & 0.765 & 0.015 \\
\hline
\end{tabular}

* Chi-square test. For Ascaris and Trichuris infections, Chi-square was not valid hence p-values not indicated

National Programme for Elimination of LF (NPELF). A previous LF research study by our team in Malindi district in the northern area of Coast Region, reported that prevalence of CFA had declined from approximately $34 \%$ to $11 \%$ after 4 rounds of MDA [15]. The results of this study suggest that LF, compared to schistosomiasis and $\mathrm{STH}$, is not a major public health problem in the study area. However, continued MDA for LF is still recommended in this setting and all other endemic areas to meet the WHO target of global elimination of the disease as a public health problem.

Other studies have shown that DEC and albendazole co-administration during LF MDA has ancillary health benefits of also controlling STH infections [16,17]. It is likely that any additional health gains in controlling $\mathrm{STH}$ made during the 3 rounds of DEC/albendazole MDA administered in Kwale district were lost when the
MDAs were missed for long periods. Reinfection with $\mathrm{STH}$ is a common problem especially in areas of high transmission.

Additionally, our findings show that malaria is also endemic in the area. Efforts should be made to integrate the often well-funded malaria programmes with specific NTDs occurring in different areas. It may be prudent to consider integration of malaria control and schistosomiasis/STH control in the study setting and other similar areas. Malaria programmes in sub-Saharan Africa are currently scaling up towards universal coverage for all populations at risk of malaria with locally appropriate interventions, including insecticide-treated nets (ITNs), so that all age groups can be protected against malaria. Addition of deworming against schistosomiasis and STH to ITN distribution campaigns in highly endemic areas would provide further health benefits for the local

Table 3 Intensities of hookworm and urinary schistosomiasis infections among adults positive for the infections in five rural villages in Kwale district, coastal Kenya

\begin{tabular}{|c|c|c|c|c|c|c|c|}
\hline \multirow[b]{2}{*}{ Infection } & \multicolumn{5}{|c|}{ Village } & \multirow[b]{2}{*}{ All } & \multirow[b]{2}{*}{$\mathbf{P}$} \\
\hline & Maponda & Mirihini & Kajiweni & Miatsani & Mlafyeni & & \\
\hline \multicolumn{8}{|l|}{ Hookworm } \\
\hline No. Positive & 40 & 58 & 64 & 44 & 29 & 235 & \\
\hline GMI EPG & 162 & 147 & 143 & 140 & 114 & 142 & 0.949 \\
\hline Light (\%) & $37(92.5)$ & $55(94.8)$ & $63(98.4)$ & $42(95.5)$ & $29(100.0)$ & $226(96.2)$ & \\
\hline Heavy (\%) & $3(7.5)$ & $3(5.2)$ & $1(1.6)$ & $2(4.5)$ & $0(0)$ & $9(3.8)$ & \\
\hline \multicolumn{8}{|l|}{ Schistosomiasis } \\
\hline No. Positive & 10 & 18 & 25 & 20 & 34 & 107 & \\
\hline GMI (eggs/10 ml urine) & 8 & 11 & 11 & 14 & 28 & 15 & 0.147 \\
\hline Light (\%) & $10(100.0)$ & 15 (83.3) & $19(76.0)$ & $15(75.0)$ & 23 (67.6) & $82(76.6)$ & \\
\hline Heavy (\%) & $0(0)$ & $3(16.7)$ & $6(24.0)$ & $5(25.0)$ & $11(32.4)$ & $25(23.4)$ & \\
\hline
\end{tabular}

Geometric mean intensities (GMI) and levels of the intensities (light or heavy) were computed for individuals positive the infections only Hookworm: light, 1-1999 EPG; heavy, $\geq 2000$ EPG.

S. haematobium: light, 1 - 49 eggs $/ \mathrm{ml}$ urine; heavy, $\geq 50$ eggs $/ \mathrm{ml}$ urine 
Table 4 Polyparasitism with urinary schistosomiasis, soiltransmitted helminths, and lymphatic filariasis among adults in $\mathbf{5}$ rural villages in Kwale district, coastal Kenya

\begin{tabular}{llllll}
\hline & & \multicolumn{4}{c}{ No. of infections (\%) } \\
\cline { 2 - 6 } Village & $\mathbf{N}$ & $\mathbf{0}$ & $\mathbf{1}$ & $\mathbf{2}$ & $\mathbf{3}$ \\
\hline Maponda & 118 & $72(61.0)$ & $35(29.7)$ & $8(6.8)$ & $3(2.5)$ \\
Mirihini & 127 & $60(47.2)$ & $54(42.5)$ & $13(10.2)$ & $0(0)$ \\
Kajiweni & 123 & $43(35.0)$ & $63(51.2)$ & $15(12.2)$ & $2(1.6)$ \\
Miatsani & 94 & $41(43.6)$ & $38(40.4)$ & $14(14.9)$ & $1(1.1)$ \\
Mlafyeni & 137 & $83(60.6)$ & $43(31.4)$ & $7(5.1)$ & $4(2.9)$ \\
All & 599 & $299(49.9)$ & $233(38.9)$ & $57(9.5)$ & $10(1.7)$ \\
\hline
\end{tabular}

communities and may be a cost-effective way of using the available resources.

The major potential weakness of this study may be the fact that only one stool sample was collected. The accuracy of the Kato-Katz technique in identifying individuals with STH infections is limited by day-to-day variation in helminth egg excretion and sensitivity is greatly reduced when intensity of infections is low [18]. Improved detection of STH eggs in stool requires examination of stool specimens collected on 2 to 3 consecutive days which may not be practical especially when working in very remote areas. A new technique known as FLOTAC has been proposed as a better tool for diagnosis of STH infections; in hookworms infection study conducted in Cote d'Ivoire, the FLOTAC technique was found to have a sensitivity of $88.2 \%$ compared with $68.4 \%$ for Kato-Katz [19]. Thus, the prevalence of STH infections, and in particular hookworms, reported in the current study may have been higher if the more sensitive FLOTAC technique was used.

It is estimated that $26-68 \%$ of individuals with schistosomiasis are carriers of an additional helminthic infection, such as hookworms, A. lumbricoides, or $T$. trichiura [20]. This study revealed that approximately $46 \%$ of adults with schistosomiasis also had hookworms. The additional parasites may aggravate morbidity due schistosomiasis infection, especially in young children [21]. Hookworm infection is a well-known cause of iron-deficiency anaemia because of intestinal blood loss. Anaemia predisposes to severe morbidity in children and pregnant women [22]. However, the majority of schistosomiasis and hookworm infections in our study were light-intensity which suggests that the effects may be quite mild.

The WHO Expert Committee on the Control of Schistosomiasis and STH during its meeting in 2001 noted that there are substantial reductions in the cost of anthelminthic drugs generally, and said that new options for treatment and retreatment strategies should be considered [2]. Since praziquantel is now available at low cost (approximately US $\$ 0.2$ per treatment) more liberalized use of the drug may become possible [23]. The results of this study suggest that it may be necessary to develop novel approaches to offer preventive chemotherapy interventions to adults in high endemic areas in an effort complement school-based deworming and to accelerate reduction of transmission of helminthic infections in such areas. Inclusion of adults in control programmes may have the potential to dramatically improve both efficiency and effectiveness of control efforts against NTDs employing integrated MDAs. It may be useful to start pilot NTDs integrated control programmes in endemic areas which may later be scaled up to regional or national programmes.

\section{Conclusions}

The findings of this study underscore schistosomiasis and STH infections as important infections among adults in areas of high endemicity for these infections. Additionally, these results suggest need for design of optimal treatment approaches that may consider including adults at risk of these infections in preventive chemotherapy interventions. Reduction of these infections in adult populations may contribute to subsequent decrease in the force of transmission in such communities leading to lower morbidities in children.

\section{Acknowledgements}

Kwale district health management team (DHMT) is thanked for its support of the research project. We would like to acknowledge the assistance of Juma Mwatasa for excellent field coordination, and laboratory staff of KEMRI and Kwale district hospital for dedication in parasitological examinations. The community members of the study villages are thanked for their cooperation in this study. Dr Patrick J. Lammie is thanked for reading earlier draft of the manuscript and offering useful suggestions. Financial support was provided by the European Foundations Initiative of Neglected Tropical Diseases (EFINTD) through a postdoctoral fellowship (Grant No. 1/84000) awarded to SMN. This paper is published with the permission of the Director, KEMRI.

\section{Author details}

${ }^{1}$ Eastern and Southern Africa Centre of International Parasite Control (ESACIPAC), Kenya Medical Research Institute (KEMRI), Mbagathi Road, Nairobi, Kenya. ${ }^{2}$ Centre for Public Health Research (CPHR), Kenya Medical Research Institute (KEMRI), Mbagathi Road, Nairobi, Kenya. ${ }^{3}$ Division of Vector-Borne \& Neglected Tropical Diseases (DVBNTD), Ministry of Public Health \& Sanitation, Nairobi, Kenya. ${ }^{4}$ Kwale District Hospital, Kwale County, Coast Region, Kenya. ${ }^{5}$ Centre for Neglected Tropical Diseases, Liverpool School of Tropical Medicine, Liverpool, UK.

\section{Authors' contributions}

SMN participated in the conception and design of the study, data collection and analyses, and drafted the manuscript. SMN, MTM and FMH supervised specimen collection and laboratory examinations. CSM participated in the conception and design of the study. SMN drafted the manuscript, whereas MJB and MTM revised it critically for intellectual content. EM helped in data management and analysis of the data. All authors read and approved the final manuscript. SMN is the guarantor of the paper.

\section{Competing interests}

The authors declare that they have no competing interests. 


\section{References}

1. Hotez PJ, Molyneux DH, Fenwick A, Ottesen E, Ehrlich Sachs S, Sachs JD: Incorporating a rapid-impact package for neglected tropical diseases with programs for HIV/AIDS, tuberculosis, and malaria. PLOS Med 2006, 3(5):e102.

2. World Health Organization: Prevention and control of schistosomiasis and soil-transmitted helminthiasis: report of a WHO expert committee. WHO Technical Report Series No 912 Geneva; 2002.

3. World Health Organization: Programme Report no. 17 - Seventeenth Programme Report of the UNICEF/UNDP/World Bank/WHO Special Programme for Research \& Training in Tropical Diseases. Geneva; 2005.

4. Michael E, Bundy DA, Grenfell BT: Re-assessing the global prevalence and distribution of lymphatic filariasis. Parasitology 1996, 112(Pt 4):409-428.

5. Muchiri EM, Ouma JH, King CH: Dynamics and control of Schistosoma haematobium transmission in Kenya: an overview of the Msambweni Project. Am J Trop Med Hyg 1996, 55(5 Suppl):127-134

6. Brooker S, Peshu N, Warn PA, Mosobo M, Guyatt HL, Marsh K, Snow RW: The epidemiology of hookworm infection and its contribution to anaemia among pre-school children on the Kenyan coast. Trans $R$ Soc Trop Med Hyg 1999, 93(3):240-246.

7. Njenga SM, Wamae CN, Mwandawiro CS, Molyneux DH: Immunoparasitological assessment of bancroftian filariasis in a highly endemic area along the River Sabaki, in Malindi district, Kenya. Ann Trop Med Parasitol 2007, 101(2):161-172.

8. Wiest PM: The epidemiology of morbidity of schistosomiasis. Parasitol Today 1996, 12(6):215-220.

9. Engels D, Chitsulo L: Schistosomiasis. In Controlling disease due to helminth infections. Edited by: Crompton D, Montresor A, Nesheim M, Savioli L. Geneva; 2003:

10. World Health Organization: Elimination of lymphatic filariaisis as a public health problem. Geneva; 1997, WHA50/1997/REC/1991.

11. Molyneux DH, Zagaria N: Lymphatic filariasis elimination: progress in global programme development. Ann Trop Med Parasitol 2002, 96(Suppl 2):S15-40.

12. Montresor A, Crompton DWT, Bundy DAP, Hall A, Savioli L: Guidelines for the evaluation of soil-transmitted helminthiasis and schistosomiasis at community level. Geneva; 1998, WHO/CTD/SIP/98.91.

13. World Health Organization: Preventive chemotherapy in human helminthiasis: coordinated use of anthelminthic drugs in control interventions: a manual for health professionals and programme managers. Geneva; 2006.

14. Miguel E, Kremer M: Worms: Identifying Impacts on Education and Health in the Presence of Treatment Externalities. Econometrica 2004, 72(1):159-217.

15. Njenga SM, Mwandawiro CS, Wamae CN, Mukoko DA, Omar AA, Shimada M, Bockarie MJ, Molyneux DH: Sustained reduction in prevalence of lymphatic filariasis infection in spite of missed rounds of mass drug administration in an area under mosquito nets for malaria control. Parasit Vectors 2011, 4(1):90.

16. Fox LM, Furness BW, Haser JK, Desire D, Brissau JM, Milord MD, Lafontant J, Lammie PJ, Beach MJ: Tolerance and efficacy of combined diethylcarbamazine and albendazole for treatment of Wuchereria bancrofti and intestinal helminth infections in Haitian children. Am J Trop Med Hyg 2005, 73(1):115-121.

17. Rajendran R, Sunish IP, Mani TR, Munirathinam A, Arunachalam N, Satyanarayana K, Dash AP: Community-based study to assess the efficacy of DEC plus ALB against DEC alone on bancroftian filarial infection in endemic areas in Tamil Nadu, south India. Trop Med Int Health 2006, 11(6):851-861.

18. Knopp S, Rinaldi L, Khamis IS, Stothard JR, Rollinson D, Maurelli MP, Steinmann P, Marti $H$, Cringoli G, Utzinger J: A single FLOTAC is more sensitive than triplicate Kato-Katz for the diagnosis of low-intensity soiltransmitted helminth infections. Trans R Soc Trop Med Hyg 2009, 103(4):347-354.

19. Utzinger J, Rinaldi L, Lohourignon LK, Rohner F, Zimmermann MB, Tschannen AB, N'Goran E K, Cringoli G: FLOTAC: a new sensitive technique for the diagnosis of hookworm infections in humans. Trans $R$ Soc Trop Med Hyg 2008, 102(1):84-90.

20. Bundy DA, Chandiwana SK, Homeida MM, Yoon S, Mott KE: The epidemiological implications of a multiple-infection approach to the control of human helminth infections. Trans R Soc Trop Med Hyg 1991, 85(2):274-276

21. Barreto ML, Loureiro S, Melo AS, Anjos CF: The effect of Schistosoma mansoni infection on child morbidity in the State of Bahia, Brazil. IIAnalysis at the individual level. Rev Inst Med Trop Sao Paulo 1985, 27(4):167-171.

22. Crompton D, Montresor A, Nesheim M, Savioli L, eds: Controlling disease due to helminth infections. Geneva; 2003.

23. Richards FO Jr, Eigege A, Miri ES, Jinadu MY, Hopkins DR: Integration of mass drug administration programmes in Nigeria: The challenge of schistosomiasis. Bull World Health Organ 2006, 84(8):673-676.

doi:10.1186/1756-3305-4-175

Cite this article as: Njenga et al:: Adult population as potential reservoir of NTD infections in rural villages of Kwale district, Coastal Kenya: implications for preventive chemotherapy interventions policy. Parasites \& Vectors 2011 4:175.

\section{Submit your next manuscript to BioMed Central and take full advantage of:}

- Convenient online submission

- Thorough peer review

- No space constraints or color figure charges

- Immediate publication on acceptance

- Inclusion in PubMed, CAS, Scopus and Google Scholar

- Research which is freely available for redistribution

Submit your manuscript at www.biomedcentral com/submit
Biomed Central 